\title{
Exploring The Role of Advanced Clinical Practitioners (ACPs) and Their Contribution To Health Services in England.
}

Claire Mann ( $\nabla$ c.mann@nottingham.ac.uk)

Nottingham University Business School University of Nottingham

Stephen Timmons

Nottingham University Business School University of Nottingham

Catrin Evans

University of Nottingham

Ruth Pearce

Head of School of Education Allied Health Professions and Midwifery University Hospitals Birmingham NHS Foundation Trust

Charlotte Overton Research

Nottingham University Business School University of Nottingham

KATHRYN Hinsliff-Smith

De Montfort University

Joy Conway

Brunel University

Research Article

Keywords: NHS, advanced clinical practitioner, interviews, barriers, facilitators, roles, advancing careers, clinician

Posted Date: July 12th, 2021

DOI: https://doi.org/10.21203/rs.3.rs-602519/v1

License: (c) (i) This work is licensed under a Creative Commons Attribution 4.0 International License. Read Full License 


\section{Abstract \\ Background}

An extended role being explored globally is that of the advanced clinical practitioner (ACP). In England this is an extended role for allied health professions, nurses and midwives. ACP education is organised by Health Education England (HEE) who organise an accredited training programme for ACPs alongside a multi-professional framework. This paper draws on research commissioned to evaluate the national implementation of the ACP role to inform the development of a Centre for Advancing Practice in the UK but has implications for others considering their ACP provision. This paper focuses on three key research questions: 1) What is the role of ACPs in England? 2) What are the barriers and facilitators to implementing the role? and 3) What is the contribution of ACPs to health services in England?

\section{Methods}

Semi-structured interviews were conducted in 2020 with 63 participants across a range of stakeholder groups and clinical areas. A purposive snowball sampling technique was used to identify participants meeting the inclusion criteria. Interviews were recorded and transcribed verbatim, anonymised and thematically analysed.

\section{Results}

We report on three aspects as highlighted from the data (1) The role of ACPs, (2) Barriers to and facilitators of the ACP role implementation (3) Contribution of ACPs to health services in England. The ACP role in England was undertaken in a broad range of clinical contexts. ACPs worked towards being an autonomous practitioner and most were independent prescribers. In England 'advanced clinical practitioner' was not a protected title. There were high levels of variability and ambiguity of understanding and deployment of the ACP role in England.

\section{Conclusions}

This study outlines the contribution that ACPs make to health services and contributing factors to the success of the ACP role in England. We demonstrated the range in which the role is successful in England. We identified key barriers and facilitators to implementing this role. Finally, the work showed the positive contribution that ACPs can make to service redesign and workforce development as well as patient outcomes, whilst accepting that there is much work to do to ensure protected status and parity across all professions and clinical contexts.

\section{Background}

Globally, all medicine and health professions are dealing with a growing supply-demand imbalance. Increasing ageing populations, and workforce shortages are adding pressure to the global health environment ${ }^{1-4}$. The recent added weight of the Covid-19 global pandemic has made the situation more acute ${ }^{5}$. There is evidence of significant shortages of GPs and emergency care clinicians in the UK National Health Service (NHS) ${ }^{4}$. Models of workforce delivery in healthcare have evolved into multidisciplinary teams (MDTs), making the most of a wide range of professional expertise. Professionals from a wide range of backgrounds are encouraged to work at an advanced level in a range of global settings ${ }^{6-15}$. One new extended role being explored globally is that of the advanced clinical practitioner (ACP). In England the ACP is an extended role for nurses and allied health professions ${ }^{2}$. In the UK, the role of advanced practice has evolved organically until quite recently, led mostly by nurses working in advanced roles ${ }^{16,17}$. In many UK allied health professions (AHPs) there have been limited opportunities to progress to an advanced level clinically until the development, recognition and certification of the ACP role. Recent policy developments are reflected in the NHS People Plan ${ }^{3}$ which has a specific commitment to new ways of working by 'making effective use of the full range of our people's skills and experiences'p3.

The ACP role is overseen and supported by Health Education England (HEE), a public body, with responsibility to the UK Government Department of Health and Social Care. HEE's aims are closely aligned to those of the NHS and have an important role to play in in providing educational pathways and multiprofessional frameworks for a cross-section of ACPs in England ${ }^{3}$. Learning for ACPs is organised by Health Education England (HEE) who have developed an accredited training programme for ACPs alongside a multi-professional framework as guidance for the role, its scope and to inform education. The aim for ACPs is to develop as autonomous practitioners confident in complex decision making The framework links clearly to the four pillars of advanced practice clinical, management and leadership, education and research ${ }^{3}$. HEE are developing a Centre for Advanced Practice to regulate educational provision, facilitate networking and sharing good practice.

In England, 'trainee ACPs' undertake masters level study and normally undertake modules including history taking, physical examination, clinical decision making, non-medical prescribing (if appropriate) then complete workplace based education/competencies under the observation of a clinical supervisor. On graduation they transition into the job title of ACP. Within England the recognition for this professional qualification can lead to change in scope and remit of role, higher clinical and management responsibilities, staff responsibilities and practical acknowledgment with a change in lanyard, uniform or job title. Responsibilities of the role varied across individuals and contexts. In England one key function of the ACP role is the ability to be an independent prescriber. The ACP role is not a 'protected' job title. 
This paper draws on research commissioned by HEE for England and reported on in $2020^{18}$. The objective of the research was to evaluate the national implementation of the ACP role from multiple stakeholder perspectives to inform the development of a Centre for Advancing Practice in the UK. The research drew on a wide range of stakeholders including Higher Education Institutions (HEls) designing and delivering ACP training, ACPs both qualified and in training, employers of ACPs, supervisors of ACPs and those in national roles related to the advancement of clinical practice. This paper focuses on three key research questions: 1) What is the role of advanced clinical practitioners (ACPs) in England? 2) What are the barriers and facilitators to implementing the role? and 3) What are the contributions of ACPs to health services in England?

\section{Methods}

Semi-structured interviews were designed to explore stakeholder perspectives on the ACP role. In total 63 key stakeholders participated in a one-to-one interview during January-December 2020 with two postdoctoral research fellows (CM, CO). Due to restrictions on movements, and pressures on the NHS, all interviews were conducted remotely by telephone or online platform e.g. Microsoft Teams and lasted between 30 and 60 minutes.

A purposive snowball sampling technique was used to identify key participants meeting the inclusion criteria. The main inclusion criteria were for participants to be actively working in a role relating to ACP or ACP education in England. Participants were invited to contact the team if willing to be interviewed and all volunteer participants were provided with an information sheet explaining the study and were asked to provide consent to participate. Throughout the study the sampling approach was revisited to ensure a reasonable spread of representation across professions and roles. For example, we recognised that no dieticians or speech and language therapists had been involved in the first 50 interviews and therefore we targeted these professions to include them before data collection ceased. The following table outlines this sampling spread.

The following tables at Fig. 1 outline this sampling spread.

Although roles are categorised as discrete above, there is significant overlap, for example most employers are also practitioners, and many are also clinical supervisors.

Since the research was focused on stakeholder groups across a range of roles, the research team developed two separate semi-structured interview schedules. Two variable interview schedules were utilised focusing on the areas of specialism - for example, prompt questions for HEls and employers focused on their experience of achieving accreditation and managing ACP implementation. For the trainee ACP interviews the questions focused on their experiences of learning and undertaking the ACP role. A range of common questions and the semi-structured nature of the interviews ensured that all participants had the opportunity to fully reflect on the ACP role and contribute to the evaluation.

All interviews were digitally recorded and transcribed verbatim, anonymised and thematically analysed. The thematic analysis utilised inductive and deductive coding and was undertaken separately by two researchers $(\mathrm{CM}, \mathrm{CO})$ using NVivo 12 software comparisons for inter-coder reliability ${ }^{19}$. Team based discussions with the wider team (CE, CM, ST, RP, KHS) facilitated iterative development of the themes presented in this paper.

The study gained ethical approval from the University (NUBS REC/2019, number 201819034)

\section{Findings}

The key findings are reported in three broad themes - (1) The role of ACPs, (2) Barriers and facilitators of the ACP role and (3) The contribution of ACPs to health services in England. The first theme explores the ACP role in England and is informed largely by the initial research question related to scoping the current ACP role. The second theme focuses on the key facilitators to implementation of the role and finally, the third theme reports the stakeholder view of the impact that the ACP role has on the NHS, in relation to workforce development and patient outcomes.

\section{Theme 1 - The role of ACPs}

Data from the study confirmed that the ACP role was wide-ranging and is undertaken in community and hospital settings, care homes and community settings as well as other locations such as secure care (for example, children's homes, young offender institutions and mental health hospitals). All ACP participants, with one exception were working in direct contact with patients (the exception was a radiographer). The 63 participants in our study came from a wide range of professional backgrounds (see Fig. 1). We identified three sub-themes relating to the role of ACPs - the scope of the role, and the dichotomies of general or specialist and role or level of practice.

\section{Sub-theme: Scope of the role}

The ACP roles of the participants in our study were very wide-ranging and there was some concern from multiple participants that the ACP role is not clearly defined. ACP it is not a protected title and there was evidence of its overuse and misuse in numerous NHS Trusts and in different clinical settings. There was some tension and confusion between nursing roles, such as the Specialist Nurse Practitioner (SNP), Advanced Nurse Practitioner (ANP) and Advanced Clinical Practitioner (ACP) job titles and roles:

And currently with the models in the UK anyone can call themselves an advanced practitioner. And we've done research at this university into the misuse of the Advanced Practitioner title, which is widely misused by people who've had no education or any training at all.

Assistant Professor, HEl (Nursing) P08 
One participant described an internal study at their organisation that revealed 50 clinical posts that have ACP in their job title but none of these roles are held by clinicians who have undertaken a Masters program aligned to the HEE ACP framework. This was a source of frustration to those working in the field who suggested the ACP title needs to be protected in order to protect the reputation and validate those who are undertaking the masters level ACP programmes. A further participant demonstrated awareness of best practice from other countries (such as Australia) where the ACP role is a protected title.

\section{Sub-theme: Generalist or Specialist}

The study participants identified advanced practice as being a combination of both general and specialist skills delivered with confidence and autonomy:

The Advanced Practitioner will have that more holistic approach to the patient and should be capable of dealing with a broader set of issues, instead of being single system focused.

Trainee ACP (Nursing, Paramedic) P28

Those experienced in delivering ACP services suggested it is a balance between advanced skills and professional background:

It's not forgetting where you've come from because you're got those very specialist skills. And you'll always utilise those skills and I suppose in the areas where you're not so sure, so I don't know if I get a completely random condition in clinic, I do all the basic history-taking, the basic assessments and then because it's diet, it's nutrition and treated, I fall back on taking my dietary assessments while I'm thinking about what else do I need to do. And it's my comfort blanket I suppose.. (laughs) And I think it's an ACP is bigger than that and sometimes it's letting go of your comfort blanket.

Practitioner (Dietician) P54

Some data suggested that the ACP role is more generalist than specialist:

It's not a bad thing being a generalist is it? If you can put your hand to a lot of things, that's actually a good thing for the patient. Because you could pick up on different things. While you're might be dealing with one specific issue, but you might pick up on other things.

Deputy Workforce Lead (Non-clinical Employer) P48

\section{Sub-theme: Role or level of practice}

A dichotomy identified by participants is whether ACP defines a role or a level of practice, despite HEE being clear that the ACP framework ${ }^{3}$ relates to an advanced level of practice:

You know, they've got ACPs doing different roles in their organisation, but they don't know where to put me because they don't assume that ... because they see it as different roles rather than a level of practice.

Programme Lead, HEl (Nursing) P35

A function of the ACP role is the ability to function autonomously. For example, doctors in our study suggested that mastery of a task was achieved when a clinical presentation had been experienced multiple times and became comfortably embedded within the clinician's scope of practice:

If you're a master of a particular task you know, it's something that you ... not just necessarily a task but you know, a particular clinical presentation, something that you've seen many times before. You've seen its variants you know, you've not just seen the kind of standard presentation and it's something that you feel comfortable with. I suppose the definition of mastery is that you're almost not having to think too hard about it you know, it's something that is very clearly within your scope of practice and you feel very comfortable with it.

Clinical Supervisor (Doctor in secondary care) P62

Within the field of nursing autonomous decision-making was linked to the ability to make decisions for example, as an independent prescriber:

So, mastery is around making those clinical decisions and acknowledging that there are multiple ways to tackle problems. But to be able to rationalise which option you have chosen and for what reasons because it would be very different from one patient to another as to what approach you choose potentially.

Clinical Lead/Supervisor (Nursing) P63

This was supported by views of a physiotherapist supervisor who suggested:

When they get to that point of being able to work with undifferentiated and undiagnosed clients and to be able to recognise that, that's when they're gaining the mastery in advanced practice.

Clinical Lead/Supervisor, (Physiotherapy) P59

This research was undertaken during the Covid-19 pandemic. There was evidence that during this time ACP roles were working in a range of flexible and responsive ways to react to the pandemic.

This section has outlined high levels of variability and ambiguity of understanding and deployment of the ACP role in England.

Theme 2 - Facilitators of the ACP role implementation

Page $4 / 11$ 
This theme explores the factors acting as facilitators to implementation of the ACP role.. We identified three sub-themes relating to this, training and education, clinical supervision and organisational support.

\section{Sub-theme: Training and Education}

The training pathway is a university approved masters ACP programme aligned to the HEE framework. The pathway comprises regular modular classroom teaching and assessments, supported by an in-practice portfolio of clinical skills developed through on-the-job learning and supervision.

Due to the range of clinical professionals undertaking the ACP role there were high levels of variation in participants' previous clinical experiences and learning needs. Different professional backgrounds had variances in the amount of direct patient contact in both the pre- and post-registration training and it was suggested that a therapeutic background, for example in nursing, gave clinicians a head start on the ACP requirements compared to other AHPs, for example pharmacists and paramedics:

Students from a paramedic background, from a nursing background, from a physio background, would be very much hands-on and very much used to speaking with patients and they will have different skills in terms of the interprofessional ... or the interrelation skills to talk to patients and get their confidence. I think the pharmacists maybe struggle a little bit more with that aspect because they don't have as much hands-on with patients that in their prereg training

ACP Lecturer, HEI (Nursing) P22

The multi-professional ACP cohorts added value to the learning experience of the group. Many participants described benefits from learning in a multidisciplinary cohort:

What they bring in terms of learning within the classroom is phenomenal because the OTs and the dieticians will bring some really valuable discussion that the nurses haven't considered, likewise physiotherapists or paramedics. So, they learn from each other so much, which is just so rich.

Programme Lead, HEl (Nursing) P36

One ACP course invited a trainee ACP with expertise in mental health to co-produce and co-deliver relevant parts of the course.

English universities have the opportunity to be accredited with the Centre for Advancing Practice, through HEE. Participants suggested the accreditation process was vital to reduce variety in courses:

It will reduce the variability in students' experience and delivery. I think you know there is a sort of perception that some courses are better than others and some students are more supported than others.

Assistant Professor, HEl (Nursing) P08

Both employers and trainee ACP participants suggested that the standards were a useful measure ensuring quality in employing ACPs and facilitating their easy movement within the NHS as a transferable skill:

As an employer I would view that we need to have a level of standardisation on regulation of some form because otherwise how do we know what we're getting when we have more and more, hundreds more ACPs in the country, then how do we standardise that across organisations?

Clinical Lead/Employer (Nursing) P63

Alternate pathways to accredit existing ACPs exist through an evidence portfolio and personalised training plan. Our participants indicated that an equivalence route is a strong facilitator to enable practitioners delivering at the ACP level to accredit their expertise and provide recognition:

There's also a mass of advanced practitioners potentially who are out there you know, who have not done a course or have part done a course, yet who are functioning and really need to be drawn in.

Programme Director, HEl (Nursing) P07

As evidenced above, multiple HEls pointed out that it would be disheartening for ACPs to be required to undertake Master's training if they could evidence working at the level required for an ACP in other meaningful ways. Indeed, some participants suggested they would pursue an equivalence route of accreditation if it was comparable.

This theme highlights that a standardised education pathway can be a facilitator to the scheme. However, the lack of protection for the role and some variances in experience could be a barrier to the scheme. Overall, through the training and education provision, HEE and the Centre for Advancing Practice play a significant role in facilitating the development of ACPs in England.

\section{Sub-theme: Clinical supervision}

Mentoring and supervision were identified as a vital component of the ACP role development and facilitator of scheme implementation and role development. The role of the clinical supervisor was twofold - supporting learning and assessing development. One participant informed us that in primary care both clinical supervision and pastoral mentoring are both offered. Some local programmes offer peer support mechanisms to underpin clinical supervision along with a pastoral support network. In two clinical settings associate supervisors were offered to underpin learning in the pillars beyond clinical - for example a research mentor appointed from the University: 
We are looking at the associate supervisor ... across the other pillars who's best-placed to supervise that ACP around those other pillars during training when they've got set goals they have to meet within the academic programme but also once qualified.

ACP Clinical Lead/Supervisor (Nursing) P58

All participants alluded to the fact that learning was not knowledge alone but the application of theory into practice. It was suggested that ACP learning was best facilitated in the workplace when learners had the opportunity to link theory and practice:

It should all be about applying things in real-life work settings and having that you know, vocational approach rather than a hugely academic sitting in a classroom and learning lots of theory.

Head of Assessment and Credentialing (Pharmacy) P61

Several participants who were supervisors pointed to the importance of shared context, in order to facilitate learning opportunistically. One supervisor suggested that direct clinical supervision of new skills and experience was essential for patient safety. A nurse supervisor suggested that the clinical supervisor needed a knowledge base of the likely issues that a learner might experience. Through revisiting particular presentations, the supervisors scaffolded complexity. One medical supervisor suggested that the best feedback for learning was when a supervisor witnessed a learner complete a clinical task repeatedly, offering reinforcement of learning with hints for improvement. Participants agreed that supervisors must strategically reduce the scaffolding to support autonomous decision making:

Everything has to be grounded in that individual scope of practice, otherwise the risk is that the advanced practice attributes are abstract and free-floating and not actually of patient care and clinical value or necessarily supporting safe care.

Director of Education (Optometry) P60

Clinical supervision was an important facilitator of the role but implemented inconsistently and there was evidence of a huge variance in experiences. Some work environments, in particular non-hospital settings, struggled to release time to provide mentoring opportunities for their ACPs:

So, if I'm working in a ward for example, the consultant will only be on there for 10 minutes and you won't see them for the rest of the day. Trainee ACP (ODP) P48

There was variance clinical supervision across masters programmes - one university provider mandated participants to spend one day per week with a clinical supervisor in contrast to another who had no specific supervision time for their ACP learners. Participants suggested that experienced ACPs could be utilised to provide effective supervision as the critical mass of ACPs develops.

Clinical supervision is a crucial facilitator to ACP development. Good quality supervision in shared contexts scaffolds learners to achieve their potential and the scaffolding can be reduced as the learner achieves autonomy. There were variances according to the support and commitment afforded to clinical supervisors by their organisation.

\section{Sub-theme: Organisational support}

Previous sub-themes outlined the importance of support for individuals, which was facilitated by support at organisational and strategic levels. Time release varied for the participants in our study, for example, one NHS Trust provided ACPs with a minimum of 6 hours per week for Continuing Professional Development (CPD). Another Trust was increasing release time for ACPs up to $10-15 \%$ of an individual's contracted hours.,

Key individuals played a key role in facilitating the role in organisations, especially at the highest level in NHS Trusts:

There needs to be really within an organisation a very clear sense of, we want ACPs and this is what we want them to do. ACP Clinical Lead/Supervisor (Nursing) P58

ACP development was reliant on the support of management at all levels and buy-in at organisational level was essential to progress the role. There was evidence of success when there was joined-up working between the HEl provider, the local trust and ACP trainees:

You know their Medical Director is on board with the ACP, he's involved with the assessments of ACP. They have real buy-in from the medical school, they have a real joined-up way of thinking around it. And we are the entire polar opposite here.

Clinical Lead/Supervisor (Nursing) P63

There was also evidence that where support or buy-in to the ACP scheme was sparse, at either individual or organisation level, this acted as a barrier to the role being implemented and benefits of the role realised:

I think if an employer is very much involved with ACPs then they probably are aware of it. But I haven't heard anything other than you know a vague sort of interest in it.

Senior Lecturer, HEI (Nursing) P09

\section{Theme 3 -The contribution of ACPs to health services in England}

This theme relates to the contribution of ACPs and the work of the NHS in two key sub-themes - the impact of individual ACPs on patient outcomes, and the impact of the role on workforce development. 


\section{Sub-theme: Patient outcomes}

We interviewed across a wide range of health professions and so were able to see the reach of the ACP role and impact of this role on patient outcomes. Participants talked about their own experiences of providing improved care for specific conditions such as Parkinson's and with stroke survivors as well as across secondary care and in community settings. In addition, participants working in other settings such as outpatient services, mental health provision and general practice (GP) shared similar stories and experiences.

There were multiple examples where the ACP made a significant impact on patient care, utilising their professional background with their new advanced clinical skills. Specific examples included a speech and language therapist who set up a triage system for patients referred into hospital at risk of laryngeal cancer to ensure they were seen within two weeks. Several pharmacists had set up schemes to support and manage specific patient groups, such as those with multimorbidities, long-term conditions or risk of falls in primary care. An occupational therapist (OT), alongside other cardiology ACPs, set up and ran a one-stop shop of follow up for heart failure patients. A dietician set up telephone consultations to start treatments for patients at home that reduced attendance at emergency care by $20 \%$. There was significant evidence from our data that showed the way in which the ACP role had a positive impact on service provision across a wide range of clinical contexts. One employer gave an example of the ACP reducing the average length of stay in hospital from 9.7 days to 2.3 days, and pointed to the financial tariffs which meant his team benefitted from $£ 500,000$ additional saving.

\section{Sub-theme: Workforce development}

In this sub-theme we report on the impact of the ACP role on workforce development. Many of our ACP participants were in roles designed strategically by the employer to fill a need and therefore fulfilling a specific workforce gap. There was also evidence that AHPs were undertaking roles which would historically have been assigned to a nurse or junior doctor, and therefore highlighting the changing roles of traditional roles and duties. In some cases, ACPs had filled a gap that was not able to be filled in any other way, clearly meeting workforce needs. Participants in all professions reported that that whilst the ACP role 'filled a gap' it did so in a way that also added value, by utilising the specific expertise of professionals, providing holistic care and freeing up doctors to focus on the specific tasks which required their attention:

We know that there are like globally a shortage of doctors, shortage of nurses, shortage of other ... but I do think that the ACPs do bring something else to the table. They do bring that holistic approach to patient care. They're not just looking at a diagnosis, a treatment and then sending the patient on their way, they are looking at the whole picture. I do think that you know they are very much keen and enthusiastic to give that extra to patients, they feel that they can do the whole journey with the patient that includes the socioeconomic kind of care as well as the sort of practical care. And I think they get to think about the impact of the condition and the disease on a patient rather than just this is your diagnosis you know, you go on and do ... now they think more broadly really around how that will impact on the patient.

Senior Lecturer, HEl (Nursing) P02

Employers and clinicians in the study gave examples of the ability to diversify the delivery of healthcare services by deploying ACPs in their clinical settings. There were several specific examples of how the role of ACP contributed to changing workforce development in departmental contexts. These include several examples from mental health services and from secondary and community care provision:

Having that baseline knowledge and understanding really enhances then their ability to, in fact, also do their mental health assessment, to really enhance their ability to prescribe. Because you suddenly have much more awareness of some of the interactions and issues and all of that. So, I think they were the first ones who really start to think, wow, this could be fantastic, and this could see us really operating in a different way, doing different things than traditional mental health nurses have done. And gradually I think other people started to get that as well

Deputy Workforce Lead (Non-clinical Employer) P48

One employer discussed that orthopaedic ACPs were able to go in to ED to assess and deliver interventions which could avoid a patient admission or reduce time required for an acute bed. There was some evidence of regional variances in workforce development in England. It is suggested that areas in the North and Midlands were leading developments which the South are slightly later to develop. There was also an indication of workforce development variances in primary and secondary care provision. There was some suggestion that there was a focus on secondary over primary care in the UK. It was suggested that ACPs can contribute to new ways of working in primary care, that will require some level of system change.

It is clear from our interview data that the ACP role can contribute to workforce development. In particular, practitioners and employers drew attention to the scope of the post to be a co-ordinating force that can focus on individualised care and responsibility for ensuring the delivery of fully integrated and meaningful care going forwards.

\section{Discussion}

Our research exploring the ACP role raised a number of key and relevant findings for a global audience. These findings relate to the role of ACPs, facilitators to implementing and developing the ACP role and the contribution of ACPs to health services.

\section{The role of ACPs}

A systematic review of the changing skill mix in the UK nearly twenty years ago demonstrated the start of a fundamental change, predominantly led by nurses but with support from AHPs ${ }^{20}$. A more recent systematic review of the ACP role ${ }^{2}$ highlighted the extent of the development of the ACP role across professions in the UK since the original work published in $2004^{20}$. By contrast a limited number of studies were identified examining a multi-professional ACP role within the global context ${ }^{6,7}$. 
Our study showed the contribution of a wide range of professions and there were limited studies which report on advanced practice across professions on a national or global level ${ }^{10,21}$. Ladd et al ${ }^{22}$ presented a global SWOT analysis of advanced practice but with sole reference to one profession, nursing. This is underpinned by a wide range of studies on advanced nursing practice across the globe ${ }^{20}$. Our study adds to this debate by demonstrating that in the English context, ACPs, regardless of profession, were aligned with the evidence for their place and value within the current and predicted demands of healthcare provision, inclusive of the current pandemic context.

The findings of this study were largely mirrored across a range of studies in the UK ${ }^{20}$. Our study demonstrates that in England, advanced clinical practice is multi-professional, is undertaken in a wide range of clinical contexts and has high levels of variability and ambiguity in understanding. There were a range of studies which consider the contribution of individual professions, such as systematic reviews of the contribution of pharmacists ${ }^{6,7}$, physiotherapists ${ }^{9}$, paramedics ${ }^{12}$ and radiographers ${ }^{13}$ adding to the studies cited in nursing ${ }^{20,22}$. The work by Evans, Poku, Pearce et al et al ${ }^{2}$ drew some interesting comparisons to our findings in reporting the breath and activity of ACP roles. This was consistent with systematic reviews of international evidence on advanced roles across settings, professions and sectors ${ }^{6-14}$.

Our study identified some problems occurring through the organic growth of advanced practice in the UK and in particular the lack of protection for the ACP title. This was consistent with published literature, for example, Sibbald, Shen \& McBride ${ }^{20}$ identified distinction between job titles and initiatives was weak, even amongst advanced nurses. Carter's scoping review ${ }^{16}$ identified similar problems in advanced practice in nursing in Canada. In more recent work Leary et al's UK study ${ }^{23}$ identified 595 different job titles being used for specialist and advanced nursing practice. Ladd et al's ${ }^{22}$ global SWOT analysis demonstrated the wide variance in protection and regulation of advanced practice in Nursing across the globe, noting a consensus model of advanced nursing practice across most of North America and Canada, and some parts of Europe, Africa and Asia. Participants in our study suggested a consensus model of advanced clinical practice cross-profession, and one participant drew attention to positive modelling in Australia. A review of advanced nursing and midwifery in Australia ${ }^{24}$ highlighted the way the role is clearly defined and understood and pointed to the benefits of this approach for the public and other healthcare providers.

ACP roles are driven to address the supply-demand imbalance, and as such it is key that they are able to practice independently and autonomously. The WHO ${ }^{1}$ outlined the importance of considering which tasks can be performed autonomously by which professionals in order to address this imbalance. Despite this intention, almost a decade later a review of skill mix in primary care across six developed nations showed limited roles beyond nursing ${ }^{25}$. Our study showed a much wider range of AHP roles in all contexts in England. The UK has the most extended nurse prescribing rights ${ }^{26}$ and widest range of non-medical prescribing professionals in the world ${ }^{26,27}$. Other countries follow closely in examining the benefits of non-medical prescribing in specific professions for example, Borthwick et al's examination of non-medical prescribing in Australasia and the UK with reference to the podiatry profession ${ }^{28}$.

Our study identified advanced practice in England as autonomous practice, usually including independent prescribing. A global scoping review of non-medical prescribing ${ }^{29}$ highlighted that only half of all countries allow non-medical prescribing and those who do often limit it to registered nurses. The Ladd et al ${ }^{22}$ global SWOT analysis of advanced nursing identified that prescribing is a developing part of the advanced nursing role in the majority of the Western world such as large parts of Northern America, Canada, Asia, Australasia, some parts of Africa and around some European countries and emerging as an important role in other areas such as Latin America/Caribbean, the rest of Africa. England leads the world on the proportion of both nurses and AHPs certified to prescribe which is a key facilitator of the multi-professional advanced clinical practice function ${ }^{26}$.

More recently, work has been conducted on the important role that ACPs are contributing to the fight against the current Covid pandemic, findings paralleled by another independent review ${ }^{5}$. Our study identified key facilitators to implementing the scheme on a national level with comparable findings from other implementation studies.

\section{Facilitating the ACP role}

Our findings indicate that education and training is an enabler to the implementation of a national ACP scheme. Likewise, Evans, Poke, Pearce et al's review ${ }^{2}$ reported on 19 papers in the UK literature which identified challenges in education and training which impacted negatively on ACP role development. The concerns identified largely related to variation in experiences, which HEE are currently addressing through the HEE accreditation process outlined in our study. Our study findings also showed that clinical supervision was an important factor to the implementation of the scheme which is a finding underpinned by the evidence presented from a range of empirical work included in Evans, Poku, Pearce et al's ${ }^{2}$ review.

Our study findings showed that organisational support can be a facilitator or barrier to the implementation of the scheme which is a finding underpinned by five studies in Evans, Poku, Pearce et al's systematic review². Our study showed differences between the uptake of the ACP role in hospital and other community organisations which are not dissimilar to reported findings in other studies both nationally and globally,8,14. One study in a community setting in the UK highlighted the difficulties associated with initiating the development of ACP roles without the organisational support of the NHS ${ }^{9}$. The authors of this paper identified the challenge of obtaining agreement for cross-sector support prohibited ACP development in the private and voluntary sectors.

\section{ACP Impact on health services}

Our study highlighted a wide range of ways that ACPs perceived their impact on patient outcomes across the NHS in England. This had striking similarities with a wide range of other studies and evidence of changing skill-mix enhancing patient outcomes in many studies. One study 20 showed that extended roles including nurse-led primary care clinics for asthma; nurse-initiated thrombolysis; nurses as patient educators in rehabilitation; and specialist home care support nurses for patients with chronic obstructive pulmonary disease or multiple sclerosis. Evans, Poku, Pearce et al's recent systematic review ${ }^{2}$ asserted 
that in the majority of UK studies, ACPs were reported to achieve positive clinical outcomes related to health status, symptoms and health behaviours. Where direct comparisons were made with medical professionals, the overwhelming majority of studies reported that ACPs achieved outcomes that were equivalent to, or better than, medical doctors ${ }^{14}$. This finding was also consistent with the findings of our study, especially data from employers in hospital settings.

Our study demonstrated that the ACPs in England contributed to a changing workforce and system redesign in line with WHO's task redesign plans ${ }^{1}$. This met the original aim of the NHS People Plan 4 , to utilise existing capacity and expertise to deliver the right care at the right time. Evans, Poku, Pearce et al's systematic review ${ }^{2}$ confirmed that UK evidence suggested that ACP roles are achieving significant benefits to patients and the healthcare system in terms of improved service accessibility, appropriateness and efficiency.

\section{Conclusion}

Overall, our work conducted in the context of implementation of ACPs in to England during 2020, outlines the contribution that multi-professionals can make to health services and some of the contributing factors to the success of the role in England thus far. We showed the scope of the role and the range of professionals and contexts in which the role was successful in England. We identified key facilitators to implementing this role nationally including the need for an accreditation training pathway, good quality clinical supervision and ongoing support at an organisational level. Finally, the work showed the positive contribution that ACPs made to service redesign and workforce development as well as patient outcomes, whilst accepting that there is much work to do to ensure protected status going forwards and parity across all allied health professions and clinical contexts.

\section{Declarations}

\section{Ethics approvals and consent to participate}

We can confirm that all participants were recruited and provided written consent to take part in line with the WMA declaration of Helsinki - ethical principles for medical research involving human subjects and agreed for publishing of data for academic purposes. The ethics approval details are NUBS REC number 201819034/2019 from Nottingham University Business School REC.

\section{Consent for publication}

All consented participants agreed for the data collected be used for the purposes of scholarly dissemination including any reports or journal submissions. All authors agreed for this manuscript to be submitted to BMC Medical Education journal.

\section{Availability of data and material}

All data generated and/or analysed during the current study are not publicly available to protect participant confidentiality but are available from the corresponding author on request.

\section{Competing interests}

We declare no competing interest in the research undertaken.

\section{Funding}

This study was funded by the Higher Education England (HEE) as a tender process and secured by a co-author Professor Stephen Timmons.

We declare no financial or non-financial competing interest for any author. Dr Catrin Evans also holds a HEE funded project that links to this programme of activity to: (1) Evaluate the Implementation and Educational Infrastructure of Advanced Clinical Practice Roles in the UK, and (2) Review the Existing Evidence Base around Advanced Clinical Practice in the UK.

\section{Authors contributions}

All authors contributed to the study conception and design. Material preparation, data collection and initial analysis were performed by authors $\mathrm{CM}$ and CO. The first draft of the manuscript was written by $\mathrm{CM}$ and all authors conducted iterative secondary analysis of data and commented on previous versions of the manuscript. All authors read and approved the final manuscript.

\section{Acknowledgments}

We gratefully acknowledge the participants for this study who freely gave their time as well as the stakeholder groups who shaped the direction of the study.

\section{References}

1. World Health Organization. (2007). Task shifting: rational redistribution of tasks among health workforce teams: global recommendations and guidelines. Available from: https://apps.who.int/iris/handle/10665/43821 (accessed 08/07/2020)

2. Evans, C., Poku, B., Pearce, R., Eldridge, J., Hendrick, P., Knaggs, R., ... Collier, R. (2020). Characterising the evidence base for advanced clinical practice in the UK: A scoping review protocol. BMJ open, 10(5), e036192.

3. Health Education England. (2017). Multi-professional framework for advanced clinical practice in England. London: Health Education England. 
4. Bailey, S. (2020) The NHS people plan.

5. HEE (2021) Analysis of the online workshop to consider the impact of Covid-19 and the implications for the future of advanced and consultant practice, accessed online 22/10/21, available online at https://www.hee.nhs.uk/sites/default/files/documents/Analysis\%20of\%20the\%20online\%20workshop\%20to\%20consider\%20the\%20impact\%20of\%20Co

6. Cardwell K \&Smith SM. (2018) Clinical pharmacists working within family practice: what is the evidence? Fam Pract. 2018;35:120 - 21.

7. Cohen V, Jellinek S, Hatch A, et al. (2009) Effect of clinical pharmacists on care in the emergency department: A systematic review. Am J Health Syst Pharm. 2009;66:1353-61.

8. Laurant M, van der Biezen M, Wijers N, et al. (2018) Nurses as substitutes for doctors in primary care. Cochrane Database Syst Rev. 2018;7:Cd001271.

9. Thompson J, McNall A, Tiplady S, et al. (2019) Whole Systems Approach: Advanced Clinical Practitioner Development and Identity in Primary Care. Journal of Health Organisation and Management. 2019;33:443 - 59.

10. Spacey A, Hipperson V, Gloster A, et al. (2020) The role of the advanced clinical practitioner in breast diagnosis: A systematic review of the literature. Radiography. 2020.

11. Weeks G, George J, Maclure K, et al. (2016) Non-medical prescribing versus medical prescribing for acute and chronic disease management in primary and secondary care. Cochrane Database Syst Rev. 2016:CD011227.

12. Bigham BL, Kennedy SM, Drennan I, et al. (2013) Expanding Paramedic Scope of Practice in the Community: A Systematic Review of the Literature. Prehospital Emergency Care. 2013;17:361 - 72.

13. Hardy M, Johnson L, Sharples R, et al. Does radiography advanced practice improve patient outcomes and health service quality? A systematic review. (2016) Br J Radiol. 2016;89:1062.

14. Martínez-González N, Djalali S, Tandjung R, et al. (2014) Substitution of physicians by nurses in primary care: a systematic review and meta-analysis. BMC Health Services Research. 2014;14:214 - 14.

15. Timmons, S., Coffey, F., Vezyridis, P. (2014) Implementing lean methods in the Emergency de[artment: the role of professions and professional status, Journal of Health Organisation and Mangement, Vol. 28 (2) pp214-228

16. Carter, N. (2010). Clinical nurse specialists and nurse practitioners: title confusion and lack of role clarity. Nursing Leadership, 189.

17. Courtenay, M., Carey, N., \& Stenner, K (2011) Non medical prescribing leads views on their role and the implementation of non medical prescribing from a multi-organisational perspective BMC Health Services Research volume 11, Article number: 142

18. Mann, C., Timmons, S., Evans C. and Pearce, R (2020) HEE Centre for Advancing Practice: Independent Evaluation Report

19. Bryman, A. and Burgess, R. G. (eds.) 1994. Analyzing qualitative data. London: Routledge

20. Sibbald, B., Shen, J., \& McBride, A. (2004). Changing the skill-mix of the health care workforce. Journal of health services research \& policy, 9(1_suppl), 28-38.

21. Saxon, R.L., Gray, M.A., Oprescu, Fl.(2014) Extended roles for allied health professionals: an updated systematic review of the evidence. J Multidiscip Healthc. 2014;7:479 - 88.

22. Ladd, E., Miller, M., Wheeler, K., Wainaina, S., Aguirre, F., McGrath, H., Lee, S., Nashwan, A., Neary, A. and Core, K., (2020). A Global SWOT Analysis of Advanced Practice Nursing: Policy, Regulation, and Practice.

23. Leary A, Maclaine K, Trevatt P, et al. Variation in job titles within the nursing workforce. J Clin Nurs. 2017;26:4945-50.

24. Lowe, G., \& Plummer, V. (2019). Advanced Practice in Nursing and Midwifery: The Contribution to Healthcare in Australia. Advanced Practice in Healthcare: Dynamic Developments in Nursing and Allied Health Professions, 51-63.

25. Freund, T., Everett, C., Griffiths, P., Hudon, C., Naccarella, L. and Laurant, M., (2015). Skill mix, roles and remuneration in the primary care workforce: who are the healthcare professionals in the primary care teams across the world? International Journal of Nursing Studies, 52(3), pp.727-743.

26. Courtenay, Molly (2019) An overview of developments in nurse prescribing in the UK. Nursing Standard, e11078.

27. Cope, LL., Aseel, SA., \& Tully, MP., (2019) Nonmedical prescribing: where are we now?, Therapeutic advances in Drug Safety

28. Borthwick, AM., Short, AJ., Nancarrow, SA. \& Boyce R, (2010) Non- medical prescribing in Australasia and the UK: the case of podiatry, Journal of Foot and Ankle Research volume 3, Article number: 1

29. Ecker, S., Joshi, R., Shanthosh, J., Ma, C., \& Webster, R. (2020). Non-Medical prescribing policies: A global scoping review. Health Policy.

\section{Figures}




\begin{tabular}{|l|l|}
\hline Professions & \\
\hline Nursing & 34 \\
\hline Pharmacy & 7 \\
\hline Paramedic & 3 \\
\hline Nursing \& Paramedic & 1 \\
\hline Non-clinical & 4 \\
\hline Physiotherapy & 4 \\
\hline Radiographer & 2 \\
\hline Speech and Language & 1 \\
\hline Radiology & 1 \\
\hline ODP & 1 \\
\hline OT & 2 \\
\hline Dietician & 1 \\
\hline Doctor & 2 \\
\hline Total participants & 63 \\
\hline
\end{tabular}

\begin{tabular}{|l|l|}
\hline Roles & \\
\hline HEI & $\mathbf{2 1}$ \\
\hline Employer & $\mathbf{1 1}$ \\
\hline Trainee ACP & $\mathbf{1 3}$ \\
\hline Practitioner & $\mathbf{1 0}$ \\
\hline Regulator & 4 \\
\hline Mentor & 4 \\
\hline
\end{tabular}

Figure 1

Breakdown of participants 